\title{
Stability of neuropsychological test performance in older adults serving as normative controls for a study on postoperative cognitive dysfunction
}

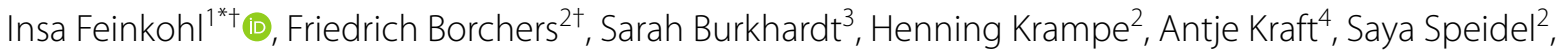
Ilse M. J. Kant ${ }^{6}$, Simone J. T. van Montfort ${ }^{6}$, Ellen Aarts ${ }^{6}$, Jochen Kruppa ${ }^{5}$, Arjen Slooter ${ }^{6}$, Georg Winterer ${ }^{7,8}$, Tobias Pischon ${ }^{1,8,9 \dagger}$ and Claudia Spies ${ }^{2 \dagger}$

\begin{abstract}
Objective: Studies of postoperative cognitive dysfunction (POCD) rely on repeat neuropsychological testing. The stability of the applied instruments, which are affected by natural variability in performance and measurement imprecision, is often unclear. We determined the stability of a neuropsychological test battery using a sample of older adults from the general population. Forty-five participants aged 65 to 89 years performed six computerized and noncomputerized neuropsychological tests at baseline and again at 7 day and 3 months follow-up sessions. Mean scores on each test were compared across time points using repeated measures analyses of variance (ANOVA) with pairwise comparison. Two-way mixed effects, absolute agreement analyses of variance intra-class correlation coefficients (ICC) determined test-retest reliability.
\end{abstract}

Results: All tests had moderate to excellent test-retest reliability during 7-day (ICC range 0.63 to 0.94 ; all $p<0.01$ ) and 3-month intervals (ICC range 0.60 to 0.92 ; all $p<0.01$ ) though confidence intervals of ICC estimates were large throughout. Practice effects apparent at 7 days eased off by 3 months. No substantial differences between computerized and non-computerized tests were observed. We conclude that the present six-test neuropsychological test battery is appropriate for use in POCD research though small sample size of our study needs to be recognized as a limitation.

Trial registration ClinicalTrials.gov Identifier NCT02265263 (15th October 2014)

Keywords: Cognitive ageing, Computerized testing, Neuropsychological testing, Postoperative cognitive dysfunction, Test-retest reliability

*Correspondence: insa.feinkohl@mdc-berlin.de

${ }^{\dagger}$ Insa Feinkohl, Friedrich Borchers, Tobias Pischon and Claudia Spies contributed equally to this work

${ }^{1}$ Max-Delbrueck-Center for Molecular Medicine in the Helmholtz Association (MDC), Robert-Roessle-Str. 10, 13092 Berlin, Germany Full list of author information is available at the end of the article

\section{Introduction}

Postoperative cognitive dysfunction (POCD) is a neurocognitive disorder (NCD) that affects around 10 to $38 \%$ of older adults during the first few months after surgery [1] but despite attempts at consensus [2] it is poorly defined. As of today POCD remains a research diagnosis that is dependent on formal, repeat neuropsychological testing rather than clinical diagnosis. Distinction of a clinically relevant cognitive change due

(c) The Author(s) 2020. This article is licensed under a Creative Commons Attribution 4.0 International License, which permits use, sharing, adaptation, distribution and reproduction in any medium or format, as long as you give appropriate credit to the original author(s) and the source, provide a link to the Creative Commons licence, and indicate if changes were made. The images or other third party material in this article are included in the article's Creative Commons licence, unless indicated otherwise in a credit line to the material. If material is not included in the article's Creative Commons licence and your intended use is not permitted by statutory regulation or exceeds the permitted use, you will need to obtain permission directly from the copyright holder. To view a copy of this licence, visit http://creativeco mmons.org/licenses/by/4.0/. The Creative Commons Public Domain Dedication waiver (http://creativecommons.org/publicdomain/ zero/1.0/) applies to the data made available in this article, unless otherwise stated in a credit line to the data. 
to surgery from natural variability and measurement error is imperative in these settings. Practice effects, for instance, lead to improved performance (or a milder decline) due to familiarity with test stimuli and testing situation [3]. To this end, rather than relying on raw cognitive change [4], surgical patients' scores are typically converted using 'reliable change index' (RCI) algorithms. These algorithms compare patients' preto post-surgery change to that of a non-surgical agematched control group [5].

There is substantial variation in the number and types of neuropsychological tests that have been used in POCD research, however [6]. This hampers comparability between studies and may account for inconsistent results in POCD incidence [7] and epidemiology [8]. Specifically, a priori evaluation of their psychometric properties including their stability over time as measured by test-retest reliability in relevant control samples is rarely considered in test selection. One previous study assessed the neuropsychological test battery of the International Study of Post-Operative Cognitive Dysfunction cohort (ISPOCD), one of the most influential studies on POCD, and found that test-retest reliability was unsatisfactory for several of its subtests [9] but these types of findings have generally been overlooked.

POCD research has also undergone a shift from conventional to computerized testing (e.g., [10]). Yet studies of POCD focus on older adults who are prone to computer anxiety [11] which may affect computerized test performance $[12,13]$. Even in younger adults, one study found only modest correlations of computerized performance with conventional, non-computerized tests [14] and in a study of older surgical patients, POCD defined from computerized tests showed only moderate agreement with POCD defined from conventional tests [15].

In sum, what is needed is a strategic evaluation of computerized and non-computerized neuropsychological tests that are commonly used in POCD research in terms of their stability over time in individuals who do not undergo surgery and thus are not expected to present with cognitive decline during a brief follow-up period. This will help investigators refine their choice of neuropsychological tests and understand methodological limitations when reporting on POCD.

Here, we determined the stability of a set of six neuropsychological tests (four computerized; two non-computerized) in a sample of older communitydwelling non-surgical controls recruited for the Biomarker Development for Postoperative Cognitive Impairment in the Elderly (BioCog) study [16]. Additionally, to help clinicians gauge whether a patient's change in test performance likely reflects a clinically relevant change, we calculated the 'smallest real difference' as the smallest within-person change that can be interpreted as a 'real' change exceeding natural variability [17].

\section{Main text \\ Study design}

We recruited a sample of older adults at outpatient clinics, primary care facilities, care homes and at public talks in Berlin, Germany, and Utrecht, the Netherlands, to serve as non-surgical control participants for the BioCog study [16]. Participants were eligible to participate if they were $\geq 65$ years old, had not undergone surgery during the past 6 months, and were not scheduled for surgery within the next 3 months. Participants were excluded if they scored $\leq 24$ on the Mini Mental State Examination (MMSE) [18], had a diagnosed neuropsychiatric disorder, reported regular intake of psychotropic medication or had severe visual or auditory impairment.

\section{Neuropsychological assessment}

Six neuropsychological tests with a total of eight outcome measures were administered once at enrolment (T0) and again in identical form at 7 days (T1), and 3 months (T2) (Table 1) [19]. Four tests were part of the Cambridge Neuropsychological Test Automated Battery (CANTAB; CANTAB Research Suite, Cambridge Cognition Ltd., UK) and were performed on touch-screen electronic devices with a press pad. The Paired Associates Learning (PAL) test of visual memory involved locating a target pattern among a set of potential boxes. Outcome measure was the 'first trial memory score'. The Verbal Recognition Memory (VRM) test of verbal memory involved sequential presentation of 12 target words, followed by free immediate recall and delayed recognition from a list of 24 words after a 20-min interval. For Spatial Span (SSP), participants were to repeat an increasingly long sequence of highlighted boxes on the screen through tapping. The test assessed spatial working memory and the number of boxes that participants could track within three attempts at each level ('spatial span') served as outcome. The Simple Reaction Time (SRT) test of processing speed involved pressing the press pad in response to a stimulus. Outcome was mean reaction time across 100 trials. Additionally, for Grooved Pegboard (GP) test of manual dexterity, participants placed 25 pegs into holes on a board using their dominant hand. For Trail-Making Test-A (TMT-A) as a measure of processing speed, participants connected dots in ascending order $(1-2-3-$ 4...). TMT-B involved alternating between letters and numbers $(\mathrm{A}-1-\mathrm{B}-2-\mathrm{C}-3 \ldots)$ and tested executive function and processing speed. 
Table 1 Summary of neuropsychological tests

\begin{tabular}{|c|c|c|}
\hline Test interface & Test & Cognitive domain \\
\hline \multirow{4}{*}{$\begin{array}{l}\text { Computerized } \\
\text { (CANTAB) }\end{array}$} & Paired Associates Learning (PAL) & Visual memory \\
\hline & $\begin{array}{l}\text { Verbal recognition memory } \\
\text { (VRM)_immediate free recall/ } \\
\text { delayed recognition }\end{array}$ & Verbal memory \\
\hline & Simple reaction time (SRT) & Processing speed \\
\hline & Spatial span (SSP) & Spatial memory \\
\hline \multirow{3}{*}{$\begin{array}{l}\text { Non-comput- } \\
\text { erized }\end{array}$} & Grooved Pegboard (GP) & Manual dexterity \\
\hline & Trail-Making-A (TMT-A) & Processing speed \\
\hline & Trail-Making-B (TMT-B) & $\begin{array}{l}\text { Processing speed, } \\
\text { executive function }\end{array}$ \\
\hline
\end{tabular}

CANTAB Cambridge Neuropsychological Test Automated Battery

\section{Data analysis}

We included only participants who attended all three testing sessions for our main analysis $(n=45)$ as we deemed this type of setting most relevant to POCD research. Patients are typically tested before surgery, again upon discharge and then re-attend the clinic for a follow-up several months thereafter. Analyses comparing baseline (T0) with 3-month follow-up (T2) were repeated post-hoc for participants who had only attended T0 and T2 $(\mathrm{n}=57$; see Additional file 1). Data on either SRT or GP were missing at T1 for one participant, respectively. Data on TMT-B were missing on T2 for one participant. These participants were not excluded.

First, mean scores on each test were compared across time points using repeated measures analyses of variance (ANOVA) with pairwise comparison between time points $\mathrm{T} 0$ to $\mathrm{T} 1$ and $\mathrm{T} 0$ to $\mathrm{T} 2$. We used un-transformed data for all analyses.

We determined relative consistency of scores over time between $\mathrm{T} 0$ and $\mathrm{T} 1$, and between $\mathrm{T} 0$ and $\mathrm{T} 2$, by calculating analyses of variance intraclass coefficient (ICC) estimates and their $95 \%$ confidence intervals. We report on a mean of multiple measurements, absolute-agreement, 2 -way mixed-effects model $[20,21]$ based on the fact that this was a test-retest (rather than inter-rater) setting and we wished to generalize our results to a setting where patients are tested on multiple occasions [20].

ICC values below 0.5 indicate poor reliability, and 0.5 to 0.75 indicate moderate reliability. Values greater than 0.75 suggest good reliability, and above 0.90 are considered excellent [20].

Finally, we calculated the 'smallest real difference' (SRD) [17]. The formula estimates the range of chance variation using the standard error of measurement (SEM) derived from the standard deviation at T0 (SD) and ICC to derive the standard error of difference $\left(\mathrm{S}_{\text {diff }}\right)$ [22].

$$
\begin{aligned}
& S E M=S D \times \sqrt{1-I C C} \\
& S_{\text {diff }}=\sqrt{2 \times[S E M]^{2}} \\
& S R D=S_{\text {diff }} \times 1.96
\end{aligned}
$$

ANOVA and ICC analyses were performed using SPSS (Version 23, SPSS, Chicago, Illinois).

\section{Results}

Forty-five participants ( $\mathrm{n}=18$ from Berlin; $\mathrm{n}=27$ from Utrecht) attended all three testing sessions (Fig. 1). Participants were between 65 and 89 years old and 53.3\% were male (Additional file 1: Table S1). Educational level was relatively high with $38.1 \%$ being universityeducated. The time between baseline (T0) and 7 day follow-up (T1) ranged from 2 to 18 days (median 7; interquartile range 6-9 days) and between baseline (T0) and 3-month follow-up (T2) ranged from 82 to 164 days (median 105; interquartile range 91-119 days).

There was a statistically significant effect of time point on performance on PAL, VRM free recall and recognition, and on TMT-B (Table 2). Pairwise comparison determined that performance on PAL, VRM free recall and TMT-B significantly improved between T0 and T1 (7-day interval; Table 2). For instance, participants were on average $11 \mathrm{~s}$ faster on the TMT-B on the second testing occasion compared with the first. Scores on VRM recognition significantly declined and performance on SRT, SSP, GP and TMT-A was unchanged during this time interval. Between T0 and T2 (3-month interval), performance only improved on TMT-B. Mean performance on all of the remaining tests did not significantly change between $\mathrm{T} 0$ and $\mathrm{T} 2$ (Table 2).

ICC estimates indicated moderate to excellent reliability for each of the tests (Table 2). Relatively lowest reliability was observed for SSP (T0 to T1, ICC 0.63; T0

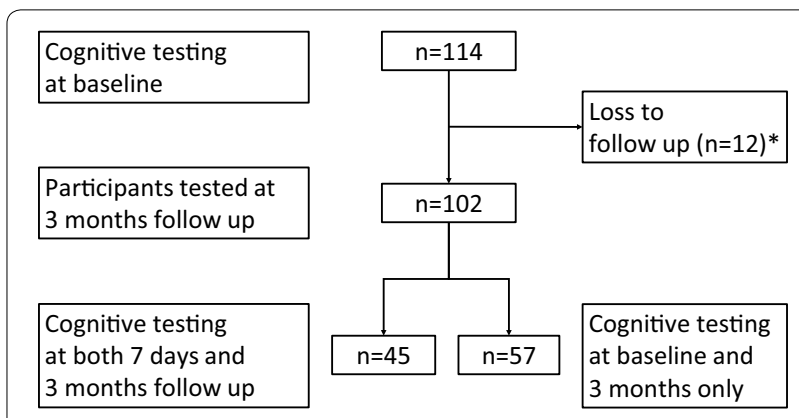

Fig. 1 Study flow chart. ${ }^{*}$ Cognitively tested at baseline $(n=5)$ or at baseline and 7 days follow up $(n=7)$ only 


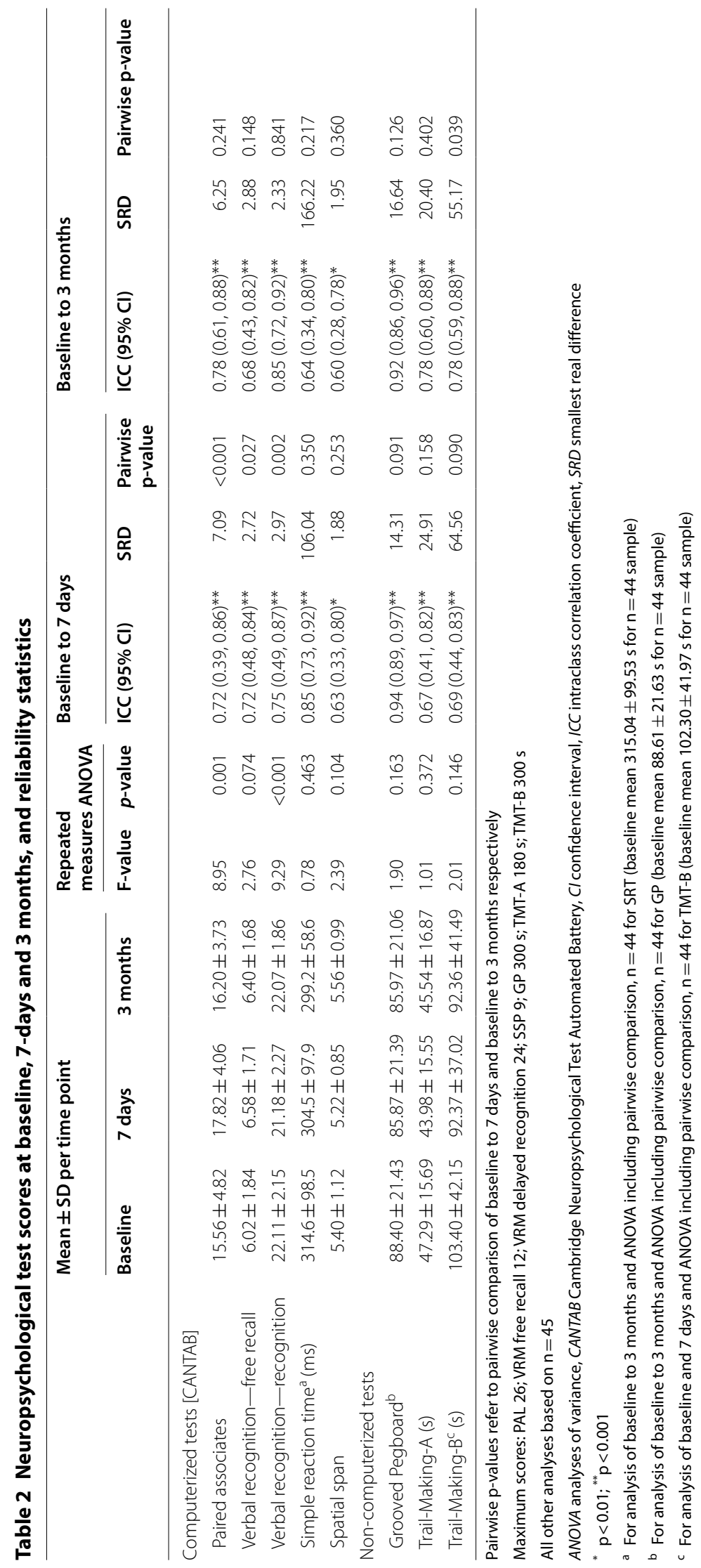


to T2, ICC 0.60). GP stood out with excellent reliability (ICC $>0.90)$ at both time intervals. Confidence intervals of ICC for GP between T0 and T1 did not overlap with any of the remaining tests except SRT indicating a statistically significantly higher ICC for GP than all other tests except SRT. ICC between T0 and T2 was also higher for GP than for VRM free recall, SRT and SSP.

'Smallest real difference' (SRD) scores are shown for each of the tests in Table 2. For instance, we found that a 166 ms increase on SRT during 3-month interval exceed natural variation and thus can be considered a relevant decline in function.

Post-hoc analysis of participants who had only attended two testing sessions $(n=57)$ revealed practice effects between $\mathrm{T} 0$ and $\mathrm{T} 2$ that were similar to those of the main analysis sample $(\mathrm{n}=45)$ for T0 to T1 though improvement was seen on different tests (Additional file 1: Table S2). In terms of ICC estimates, GP (ICC >0.90) and TMT-B (ICC 0.88) stood out with excellent and good test-retest reliabilities respectively.

\section{Discussion}

We set out to assess the stability of the BioCog neuropsychological test battery in a sample of older adults and found tests to have moderate to excellent test-retest reliability throughout. Practice effects for several tests at 7 days appeared to ease off by 3-month follow-up, despite the fact that at 3 months, participants benefited from having already been exposed to testing material and situation twice. GP stood out with excellent test-retest reliability throughout. However, GP relies heavily on motor function and hand-eye coordination [23], and so we do not recommend it as a sole indicator of neurocognitive functioning for research purposes.

Our neurocognitive test battery consisted both of traditional non-computerized and of computerized tests. Computerized testing comes with a number of advantages such as immunity to tester effects or transcribing errors. These advantages might not outweigh methodological difficulties that apply to older adults who may be affected by computer anxiety [11-13], however. Here, in line with a previous study of CANTAB [24], test-retest reliability of computerized tests was moderate to good. We found no evidence of differences in test-retest reliability between the computerized tests and the noncomputerized tests. Thus computerized tests were overall subject to no greater intra-individual variability compared with traditional tests.

For each neurocognitive test, we provided the 'smallest real difference' (SRD) $[17,25]$ to help clinicians determine whether a change in scores of a patient is likely of concern. Yet it should be noted that SRD values apply to the present sample and follow-up period only.
Future studies are advised to scrutinize the psychometric properties of neuropsychological tests prior to their application. Based on our results, we see no problem with the use of computerized tests such as CANTAB in older adults. We suggest that studies (especially those defining POCD from raw change $[4,6])$ consider skipping the respective briefest follow-up session and instead focus their efforts on subsequent follow-ups that may be less affected by practice.

\section{Limitations}

Strengths of our analysis include combination of computerized with non-computerized format. However, our sample size was small as evidenced in large confidence intervals. For instance, Simple Reaction Time showed 'moderate' test-retest reliability during 3-month interval, but 95\% confidence intervals stretched from 'poor reliability' to 'good reliability'. Follow-up periods varied between participants and their relatively high educational status limits the generalizability of our findings. Finally, readers should note that a stricter cut-off for acceptable reliability (e.g., ICC $>0.8$ ) should be preferred if neuropsychological testing is applied in a clinical rather than a research setting such as our own.

\section{Supplementary information}

Supplementary information accompanies this paper at https://doi. org/10.1186/s13104-020-4919-3.

Additional file 1: Table S1. Baseline characteristics. Table S2. Neuropsychological test scores at baseline and 3 months, and reliability statistics for patients tested at baseline and 3 months only $(n=57)$.

\section{Abbreviations \\ CANTAB: Cambridge Neuropsychological Test Automated Battery; GP: Grooved Pegboard; ICC: Intraclass correlation coefficient; NCD: Neurocognitive disorder; PAL: Paired associates learning; POCD: Postoperative cognitive dys- function; RCI: Reliable change index; SRD: Smallest real Difference; SRT: Simple reaction time; SSP: Spatial span; TMT-A: Trail-Making Test-A; TMT-B: Trail-Making Test-B; VRM: Verbal Recognition Memory.}

\section{Acknowledgements}

Not applicable.

\section{Authors' contributions}

Study concept and design: GW, AS, CS, TP. Data collection: FB, SB, SS, IK, SM and EA. Statistical analysis and interpretation: FB, SB and IF. Drafting of initial manuscript: IF and FB. Review of manuscript for critical intellectual content: IF, FB, JK, HK, AK, SB, AS, GW, CS and TP. All authors read and approved the final manuscript.

\section{Funding}

This work was funded by the European Union, Seventh Framework Programme [FP7/2007-2013], under Grant agreement no. HEALTHF2-2014-602461 BioCog [Biomarker Development for Postoperative Cognitive Impairment in the Elderly]: http://www.biocog.eu. The funder had no role in the design of this study, collection of data, data analysis, interpretation of results or writing of this manuscript. 


\section{Availability of data and materials}

The datasets generated during and/or analysed during the current study are not publicly available but are available from the corresponding author on reasonable request.

\section{Ethics approval and consent to participate}

Participants gave written informed consent to participate in the study. The study protocol was approved by the local ethics committees (Ethikkommission Charité Universitätsmedizin Berlin, Reference No. EA2/092/14 and Medisch Ethische Toetsingscommissie, Universitair Medisch Centrum Utrecht, Reference No. 14-469) and was conducted in accordance with the Declaration of Helsinki. The BioCog study was registered on ClinicalTrials.gov Identifier: NCT02265263.

\section{Consent for publication}

Not applicable.

\section{Competing interests}

GW is coordinator of the BioCog consortium and is chief executive of the company Pharmaimage Biomarker Solutions GmbH [http://www.pi-pharm aimage.com]. Among other academic and private partners, the company is a partner of the BioCog study. CS and TP are project leaders in BioCog. CS, TP, IF, $\mathrm{FB}, \mathrm{SB}, \mathrm{HK}, \mathrm{AK}, \mathrm{SS}, \mathrm{IK}, \mathrm{SM}, \mathrm{EA}$, JK, and AJ declare that they have no competing of interest.

\section{Author details}

${ }^{1}$ Max-Delbrueck-Center for Molecular Medicine in the Helmholtz Association (MDC), Robert-Roessle-Str. 10, 13092 Berlin, Germany. ${ }^{2}$ Department of Anesthesiology, Charité-Universitätsmedizin Berlin, Corporate Member of Freie Universität Berlin, Humboldt-Universität zu Berlin and Berlin Institute of Health, Berlin, Germany. ${ }^{3}$ Faculty of Psychology, Philipps-Universität Marburg, Marburg, Germany. ${ }^{4}$ Department of Psychiatry, Psychiatric University Hospital St. Hedwig, Charité-Universitätsmedizin Berlin, Berlin, Germany. ${ }^{5}$ Institute of Biometry and Clinical Epidemiology, Charité-Universitätsmedizin Berlin, Corporate Member of Freie Universität Berlin, Humboldt-Universität zu Berlin and Berlin Institute of Health, Berlin, Germany. ${ }^{6}$ Department of Intensive Care Medicine, UMC Utrecht Brain Center, University Medical Center Utrecht, Utrecht University, Utrecht, The Netherlands. ${ }^{7}$ Pharmaimage Biomarker Solutions $\mathrm{GmbH}$, Berlin, Germany. ${ }^{8}$ Charité-Universitätsmedizin Berlin, Corporate Member of Freie Universität Berlin, Humboldt-Universität zu Berlin and Berlin Institute of Health, Berlin, Germany. ${ }^{9}$ MDC/BIH Biobank, Max-DelbrueckCenter for Molecular Medicine in the Helmholtz Association (MDC), and Berlin Institute of Health $(\mathrm{BIH})$, Berlin, Germany.

Received: 1 August 2019 Accepted: 24 January 2020

Published online: 04 February 2020

\section{References}

1. Feinkohl I, Winterer G, Pischon T. Obesity and post-operative cognitive dysfunction: a systematic review and meta-analysis. Diabetes Metab Res Rev. 2016:32:643-51.

2. Evered L, Silbert B, Knopman DS, Scott DA, DeKosky ST, Rasmussen LS, et al. Recommendations for the nomenclature of cognitive change associated with anaesthesia and surgery-2018. Br J Anaesth. 2018;121(5):1005-12.

3. Wilson RS, Beckett LA, Barnes LL, Schneider JA, Bach J, Evans DA, et al. Individual differences in rates of change in cognitive abilities of older persons. Psychol Aging. 2002;17:179-93.

4. Keizer AMA, Hijman R, Kalkman CJ, Kahn RS, van Dijk D. The incidence of cognitive decline after (not) undergoing coronary artery bypass grafting: the impact of a controlled definition. Acta Anaesthesiol Scand. 2005;49:1232-5

5. Rasmussen LS, Larsen K, Houx P, Skovgaard LT, Hanning CD, Moller JT, et al. The assessment of postoperative cognitive function. Acta Anaesthesiol Scand. 2001:45:275-89.

6. Collie A, Darby DG, Falleti MG, Silbert BS, Maruff P. Determining the extent of cognitive change after coronary surgery: a review of statistical procedures. Ann Thorac Surg. 2002;73(6):2005-11.
7. Androsova G, Krause R, Winterer G, Schneider R. Biomarkers of postoperative delirium and cognitive dysfunction. Front Aging Neurosci. 2015. https://doi.org/10.3389/fnagi.2015.00112.

8. Feinkohl I, Winterer G, Pischon T. Hypertension and risk of post-operative cognitive dysfunction (POCD): a systematic review and meta-analysis. Clin Pract Epidemiol Ment Health. 2017;13:27-42.

9. Lowe C, Rabbitt P. Test/re-test reliability of the CANTAB and ISPOCD neuropsychological batteries: theoretical and practical issues. Cambridge neuropsychological test automated battery. international study of postoperative cognitive dysfunction. Neuropsychologia. 1998;36(9):915-23.

10. Shoair OA, Grasso MPI, Lahaye LA, Daniel R, Biddle CJ, Slattum PW. Incidence and risk factors for postoperative cognitive dysfunction in older adults undergoing major noncardiac surgery: a prospective study. J Anaesthesiol Clin Pharmacol. 2015;31:30-6.

11. Yoon HE, Jang Y, Xie B. Computer use and computer anxiety in older Korean Americans. J Appl Gerontol. 2016;35(9):1000-10.

12. Laguna K, Babcock RL. Computer anxiety in young and older adults: implications for human-computer interactions in older populations. Comput Hum Behav. 1997;13(3):317-26.

13. Mators Goncalves M, Pinho MS, Simoes MR. Effects of socio-demographic variables on performance on the Cambridge neuropsychological automated tests for the assessment of dementia and Portuguese norms for older adults living in retirement homes. Clin Neuropsychol. 2016:30(S1):1395-428.

14. Smith PJ, Need AC, Cirulli ET, Chiba-Falek O, Attix DK. A comparison of the Cambridge automated neuropsychological test battery (CANTAB) with "traditional" neuropsychological testing instruments. J Clin Exp Neuropsychol. 2013;35(3):319-28.

15. Radtke FM, Franck M, Papkalla N, Herbig TS, Weiss-Gerlach E, Kleinwaechter $\mathrm{R}$, et al. Postoperative cognitive dysfunction: computerized and conventional tests showed only moderate inter-rater reliability. J Anesth. 2010;24(4):518-25.

16. Winterer $G$, Androsova G, Bender O, Boraschi D, Borchers F, Dschietzig $\mathrm{TB}$, et al. Personalized risk prediction of postoperative cognitive impairment — rationale for the EU-funded BioCog project. Eur Psychiatryy. 2018;50:34-9.

17. Schougaard LMV, de Thurah A, Bech P, Hjollund NH, Christiansen DH. Test_-retest reliability and measurement error of the Danish WHO-5 Wellbeing Index in outpatients with epilepsy. Health Qual Life Outcomes. 2018;16(1):175

18. Folstein MF, Folstein SE, McHugh PR. 'Mini-mental state'. A practical method for grading the cognitive state of patients for the clinician. J Psychiatr Res. 1975;12:189-98.

19. Lammers F, Borchers F, Feinkohl I, Hendrikse J, Kant IMJ, Kozma P, et al Basal forebrain cholinergic system volume is associated with general cognitive ability in the elderly. Neuropsychologia. 2018;119:145-56.

20. Koo TK, Li MY. A guideline of selecting and reporting intraclass correlation coefficients for reliability research. J Chiropr Med. 2016;15:155-63.

21. Shrout PE, Fleiss JL. Intraclass correlations: uses in assessing rater reliability. Psychol Bull. 1979;86(2):420-8.

22. Ferguson RJ, Robinson $A B$, Splaine $M$. Use of the reliable change index to evaluate clinical significance in SF-36 outcomes. Qual Life Res. 2002;11(6):509-16.

23. Bezdicek O, Nikolai T, Hoskovcova M, Stochl J, Brozova H, Dusek P, et al. Grooved pegboard predicates more of cognitive than motor involvement in Parkinson's disease. Assessment. 2014;21(6):723-30.

24. Matos Goncalves M, Pinho MS, Simoes MR. Test-retest reliability analysis of the Cambridge neuropsychological automated tests for the assessment of dementia in older people living in retirement homes. Appl Neuropsychol. 2016;23(4):251-63.

25. Lyall DM, Cullen B, Allerhand M, Smith DJ, Mackay D, Evans J, et al. Cognitive test scores in UK Biobank: data reduction in 480,416 participants and longitudinal stability in 20,346 Participants. PLOS ONE. 2016;11(4):e0154222.

\section{Publisher's Note}

Springer Nature remains neutral with regard to jurisdictional claims in published maps and institutional affiliations. 